questionnaires of fatigue (FACIT-FATIGUE), quality of life (EQ-5D-5L), disability (HAQ) and a Global Health Status Scale (GHS) (0-100). Biostatistical analysis was performed using the multivariate analysis of variance by Pillai test.

Results 54 SLE patients $(91.84 \%$ female) with a mean age at diagnosis of $27.55 \pm 13.21$ years and a mean time of disease evolution of $20.45 \pm 9.7$ years were included. Mean SLEDAI score was $6.63 \pm 6.89$, with a $37.04 \%$ of patients with SLEDAI $>6$. The $64.66 \%$ of patients were under glucocorticoid treatment, $38.77 \%$ under immunosuppressants (methotrexate, azatioprine or mycophenolate) and $51.02 \%$ under antimalarials.

Patients showed a mean score of $34.02 \pm 12.38$ in FACITFATIGUE, $0.72 \pm 0.26$ in EQ-5D-5L, $0.62 \pm 0.71$ in HAQ and $64.02 \pm 25.93$ in GHS.

Statistical analysis showed correlation among high SLEDAI scores and low scores of EQ-5D-5L, FACIT-FATIGUE and GHS, and an increment in HAQ, considering as correcting factors the age, years of disease evolution, glucocorticoid treatment, antimalarials and immunosuppressants $(\mathrm{P}=0.0107)$.

Conclusions We observed a correlation between PROs-QL fullfilled by SLE patients with the clinical activity of the disease, independently of glucocorticoid treatment, antimalarials and immunosuppressants, the age and the disease evolution.

\section{P181 COMPARISON OF SLEDAI-2K AND SLEDAI-2KG (GLUCOCORTICOID) INDEXES IN PATIENTS WITH SYSTEMIC LUPUS ERYTHEMATOSUS (SLE)}

${ }^{1}$ Selma Sari, ${ }^{2}$ Bahar Artim Esen, ${ }^{2}$ Ahmet Gül, ${ }^{2}$ Lale Öcal, ${ }^{2}$ Murat Inanç. ${ }^{1}$ Dept. of Internal Medicine, Istanbul Faculty of Medicine, Istanbul University, Istanbul; ${ }^{2}$ Dept. of Internal Medicine, Division of Rheumatology, Istanbul Faculty of Medicine, Istanbul University, Istanbul, Turkey

\subsection{6/lupus-2020-eurolupus.223}

Background Disease activity measurement in SLE can be performed with SLEDAI based on clinical and laboratory findings. The new SLEDAI-2K-glucocorticoid index (SLEDAI-2KG) developed from SLEDAI-2K calculates disease activity by taking into account the amount of glucocorticoids used. In this cross-sectional prospective study, two indexes were compared in consecutive SLE patients.

Methods Seventy-nine SLE patients were included into the study. Disease activity was evaluated using SLEDAI-2K and SLEDAI-2KG. Patients were grouped as SLEDAI $=0$ (group 1), lupus low disease activity status (LLDAS) (group 2) and active disease (group 3). LLDAS was defined as:(SLEDAI)-2K $\leq 4$, with no activity in major organ systems and no haemolytic anaemia or gastrointestinal activity; no new lupus disease activity; PGA (scale $0-3$ ) $\leq 1$; a current prednisolone dose $\leq 7.5 \mathrm{mg}$ daily; and stable maintenance doses of immunosuppressive drugs and approved biological agents.

Results Table 1 shows clinical features of SLE patients. Eighty-six percent of the patients were female. Median age 34 (range 18-74), median disease duration 36 (0-436) months. Thirty-five percent of the patients had renal activity, $7 \%$ had malar rash, $12 \%$ had alopecia, 2 (2.5\%), $8 \%$ had thrombocytopenia, $8 \%$ had leucopenia, 3.8\% had fever. Sixtyone percent of the patients had hypocomplementemia and $29 \%$ had anti-dsDNA positivity. Glucocorticoids were used by 63 patients and the median prednisone dose was 16 (0$75) \mathrm{mg}$. The median of SLEDAI-2K score of 79 patients was 4 (range $0-24$ ) and the median of SLEDAI-2KG score was 7
Abstract P181 Table 1 Demographic and clinical characteristics of SLE patients

\begin{tabular}{ll}
\hline & SLE, n=79 \\
\hline Male/Female, & $68 / 11$ \\
Age, years (median, range) & $34(18-74)$ \\
Disease duration, months (median, range) & $36(0-436)$ \\
Manifestations & \\
Vasculitis, n (\%) & $10(12,7)$ \\
Arthritis, n (\%) & $5(6,3)$ \\
Renal activity, n (\%) & 28 \\
Proteinuria, n (\%) & 26 \\
Hemolytic anemia, n (\%) & $6(7,5)$ \\
Trombositopenia, n (\%) & $7(8,9)$ \\
Autoantibodies & \\
ANA, n (\%) & $74(93,7)$ \\
Anti-dsDNA, n (\%) & $23(29,1)$ \\
C3 levels (mg/dl, median, range) & $81(34-185)$ \\
C4 levels, (mg/dl, median, range) & $2(2-42)$ \\
SLEDAl score (median, range) & $4(0-24)$ \\
SLEDAI-2KG score (median, range) & $7(0-25)$ \\
Treatment & \\
Prednisone, n (\%) & $63(79,7)$ \\
Azathioprine, n (\%) & $21(26,6)$ \\
Antimalarial, n (\%) & $52(62,8)$ \\
Mycophenolate, n (\%) & $21(26,6)$ \\
\hline & \\
\hline
\end{tabular}

(range 0-25). Significant positive correlation was found between SLEDAI-2K and SLEDAI-2KG scores $(r=0,93$, $\mathrm{p}<0,01)$. When SLEDAI-2K and SLEDAI-2KG were compared, the proportion of patients with disease activity 0 was $24 \%$ and 9\%, LLDAS $20 \%$ and $27 \%$, and active patients $56 \%$ and $64 \%$, respectively.

Conclusion Although there was a significant correlation between SLEDAI-2K and SLEDAI-2KG, more patients were defined as active with SLEDAI-2KG. Considering the importance of reducing glucocorticoid dose in clinical trials in the assessment of treatment response, SLEDAI-2KG may provide a more precise treatment response. Prospective studies are required to investigate the importance of SLEDAI-2KG in long-term prognosis of SLE patients.

\section{P182 ORGAN DAMAGE IN CROATIAN COHORT OF PATIENTS WITH CHILDHOOD ONSET SYSTEMIC LUPUS ERYTHEMATOSUS}

${ }^{1}$ Nastasia Kifer, ${ }^{1}$ Mario Sestan, ${ }^{1}$ Emilija Hosticka, ${ }^{1}$ Maja Novoselec, ${ }^{1}$ Mateja Batnozic Varga, ${ }^{1}$ Ivan Padjen, ${ }^{1}$ Marijan Frkovic, ${ }^{2}$ Domagoj Kifer, ${ }^{1}$ Branimir Anic, ${ }^{1}$ Drago Batinic, ${ }^{1}$ Kristina Potocki, ${ }^{1}$ Ivan Malcic, ${ }^{1}$ Marija Jelusic. ' University of Zagreb School of Medicine, Zagreb; ${ }^{2}$ Faculty of Pharmacy and Biochemistry, University of Zagreb, Zagreb, Croatia

\subsection{6/lupus-2020-eurolupus.224}

Background Our aim was to explore the correlation between the Systemic Lupus Erythematosus Disease Activity Index (SLEDAI 2K) at the time of diagnosis and the SLICC/ACR damage index (SDI) of patients at their last follow up, to examine organ damage and to predict the risk of organ damage occurrence in time.

Methods The retrospective study included children with childhood onset systemic lupus erythematosus (cSLE) treated from 1991 to 2017 at University Hospital Centre Zagreb. 